\title{
LUT
}

University

\section{Demonstrating the Impact of LTE Communication Latency for Industrial Applications}

\author{
Polunin Fedor, Carrillo Melgarejo Dick, Lindh Tuomo, Pinomaa Antti, Nardelli \\ Pedro H. J., Pyrhönen Olli
}

This is a Post-print

version of a publication

published by IEEE

in 2019 IEEE 17th International Conference on Industrial Informatics (INDIN)

DOI: $\quad 10.1109 /$ INDIN41052.2019.8972105

Copyright of the original publication: (C) 2019, IEEE

Please cite the publication as follows:

F. Polunin, D. C. Melgarejo, T. Lindh, A. Pinömaa, P. H. J. Nardelli and O. Pyrhonen, "Demonstrating the Impact of LTE Communication Latency for Industrial Applications," 2019 IEEE 17th International Conference on Industrial Informatics (INDIN), Helsinki, Finland, 2019, pp. 977-982. doi: 10.1109/INDIN41052.2019.8972105

(C) 2019 IEEE. Personal use of this material is permitted. Permission from IEEE must be obtained for all other uses, in any current or future media, including reprinting/republishing this material for advertising or promotional purposes, creating new collective works, for resale or redistribution to servers or lists, or reuse of any copyrighted component of this work in other works.

This is a parallel published version of an original publication. This version can differ from the original published article. 


\title{
Demonstrating the Impact of LTE Communication Latency for Industrial Applications
}

\author{
Fedor Polunin, Dick Carrillo Melgarejo, Tuomo Lindh, Antti Pinomaa, Pedro H. J. Nardelli, Olli Pyrhönen \\ School of Energy Systems \\ LUT University \\ Finland
}

\begin{abstract}
This paper assesses the performance of a private LTE wireless network in an industrial automation setup. Our goal is to study the impact of communication latency in a robot arm position control application when the position feedback signal is transmitted via a commercial LTE wireless link. An Ethernet link scenario is considered as our benchmark. We show that even under very specific conditions (no network load, high signal power), the wireless link cannot achieve a satisfactory performance. The proposed demonstration provides a simple visualization of how LTE network latency disturbs the robot behavior, leading to undesired overshoots, oscillations and even stopping its movements. These results demonstrate that even a private LTE network is still incapable of providing the low latency required by industry automation applications, which shall be incorporated in the upcoming $5 \mathrm{G}$ wireless systems.

Index Terms-LTE, latency, industrial automation, IIoT
\end{abstract}

\section{INTRODUCTION}

The latest advances on information and communication technologies (ICTs) are transforming towards industrial processes. This tendency is evinced by the term industrial internet, in which both the public and private industrial sector foresee many economic benefits [1]. It is envisioned that the production line will be optimized with better management, energy efficiency, product planning, and fault and resource prediction [2]. More specifically, wireless technologies are further enabling different classes of industrial cyber-physical systems (ICPS) where automated systems rely on the wireless connectivity to operate physical reality via computing and communication infrastructures [3]-[5].

Discussions about Industrial Internet of Things, Industrial Cyber-physical systems and Industry 4.0 are now widespread, as discussed in a dedicated special issue of IEEE Communications Magazine [6]. It is remarkable, though, that feedback control applications require extremely low latency and high reliability from any communication system. These requirements are usually hard conditions that the system must fulfill to operate correctly [7]. Wireless communication for the demands of industry applications, in its turn, is being developed in a very high pace. New standards and techniques are promising to achieve that level of performance. In this context, wireless becomes an important enabler of automation technologies.

It is expected that fifth generation of mobile network (5G) will be available in the upcoming years to support a diversity of applications including high data rate, massive MachineType Communication (MTC), and Ultra Reliable Low Latency
(URLL) communication [8]-[10]. Wireless engineers and academics usually assure that latency as low as tens of milliseconds can be already achieved with commercial solutions [11], like 4G Long-Term Evolution (LTE) technologies. Automation engineers who work in the field and industrial plants, on the other hand, are reluctant to switch the cable connections to wireless ones. This paper tries to contribute to this discussion by showing assess the performance of a private, commercial, LTE network available for industrial applications.

Latency in LTE is a byproduct of the system architecture, which is based on the system architecture evolution (SAE) or LTE/SAE architecture [12]. This contains two main evolved subsystems: evolved UTRAN (E-UTRAN) and evolved packet core (EPC) [13]. Both of these are described in Fig. 1 and summarized next. The EPC is also know as core network. It can be connected to other 3rd Generation Partnership Project (3GPP) and non-3GPP radio-access networks. The EPC consists of one control-plane node, called mobility management entity (MME), and two user-plane nodes, called serving gateway (S-GW) and packet-data network gateway $(\mathrm{P}-\mathrm{GW})$ [14]. The other subsystem is the E-UTRAN, which is represented by base stations or evolved NodeB (eNodeB), that are connected to each other through the X2 interface and to the EPC through the S1 interface [15]. The LTE radio access network is the main function of the E-UTRAN. It considers orthogonal frequency-division multiple access (OFDMA) as multiple access technique on Orthogonal Frequency Division Multiplexing (OFDM), which is a widely known waveform used in many wireless technologies. All aforementioned subsystems contribute to full network LTE latency, and we aim to analyze by using a commercial wireless connectivity setup claimed to support a real industry application.

Fig. 2 shows a theoretic latency expected in radio air interface for LTE Rel. 10 [16]. The expected round trip time (RTT) between one user equipment (UE) and one eNodeB is approximately $8 \mathrm{~ms}$. Then, if data is transmitted between two UEs, the total RTT is duplicated. Considering the delay added by the core network, this value is typically more than $20 \mathrm{~ms}$ for a full network (E-UTRAN and EPC). This delay could be increased when the error probability of the first hybridautomatic repeat request (HARQ) retransmission is more than $10 \%$ block error rate (BLER) [17], [18]. In other words, if any impairment that affects to the BLER is inserted, the number of HARQ will be increased, and as a consequence the latency 


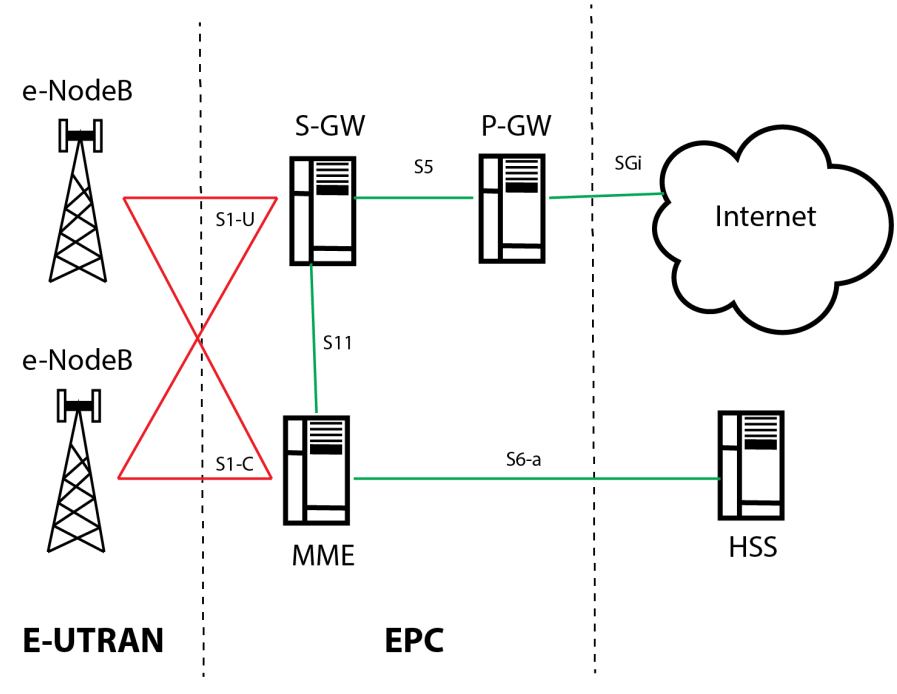

Fig. 1. LTE Architecture based on SAE. It is composed by two main elements: E-UTRAN and EPC.

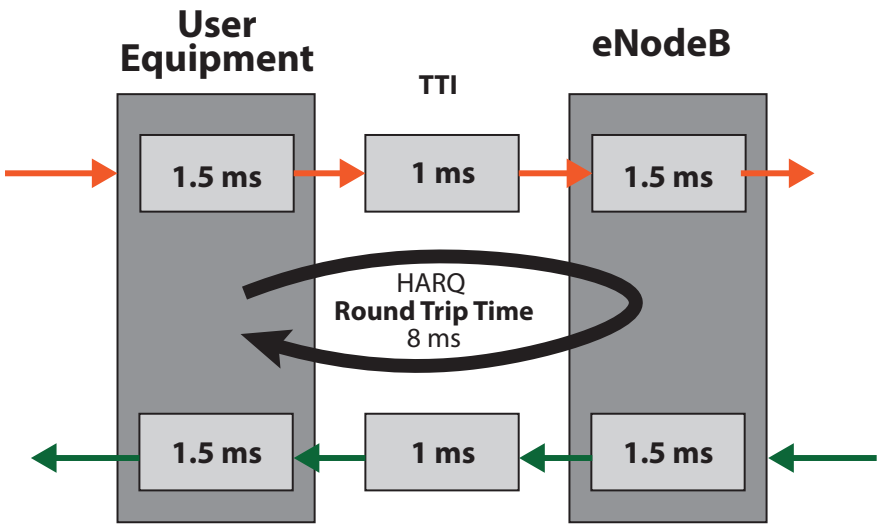

Fig. 2. Theoretic User plane latency components expected in the LTE radio interface [16]

will also be increased.

Our results show that under a very specific test environment - no impairments, low signal attenuation, no additional network load - the full network LTE link can achieve an average latency as low as $26 \mathrm{~ms}$ (what is predicted by the standard). This leads to poor performance in the movement control task. However, this communication latency grows even more under other, not so perfect conditions.

This paper shows the impact of this latency by analyzing the robot arm position overshoots and oscillations, even making the machine to stop. Therefore, depending on magnitude of the latency, possible outcomes vary from a stable transient with or without overshoot to completely unstable oscillating transient. Hence, technological process requirements to position control quality must be taken into account while determining possible latency boundaries. This result indicates whether a LTE network can be used or not. The closed-loop position control application is used in this paper to analyze its sensitivity to feedback signal latency using a real robot arm.

The rest of the paper is divided as follows. Section II presents the system model and the methodology used in this study. Section III describes the experiments used in this paper and their respective numerical results, and discusses the results got. Finally, Section IV concludes this paper.

\section{SYSTEM MODEL AND METHODOLOGY}

Our system model is based on the description of a private LTE network for industrial automation as described by 3GPP [19] (see Fig. 3). To determine the latency introduced by LTE network, a cartesian pick-and-place robot $Y$-axis position control application is used, as shown in Fig. 4. This robot is controlled by an Industrial Personal Computer (IPC) that handles position control for each robot axis as well as multiple other tasks. The idea is to route $Y$-axis position feedback signal through the private LTE network. Position measurement is carried out by a linear encoder, and then processed by two different encoder modules. To implement position measurement transmission through LTE network, a second IPC was introduced, and one encoder module was connected to each of the IPCs. Then, measurement obtained by the second IPC is sent over LTE network to the first one. At the same time, first IPC gets position measurement from its own encoder module. Finally, two measurements are compared and latency value is derived. It also has to be mentioned, that in experiments presented below, User Datagram Protocol (UDP) was used for measurement transmission.

To support our study, we used the architecture defined by 3GPP in [19]. One important definition is the utilization of dedicated local EPCs and dedicated local random access networks (RAN) (eNodeBs) to support specific industrial automation applications. In Fig. 3 it is possible to visualize a system architecture that represents a factory floor that adopts a cellular technology solution, where a variety of devices, machines, sensors, actuators and robots are communicating to coordinate and share data. It should be mentioned here that a similar approach was considered in the configuration that was considered in this study. Thus, the resulting test setup is presented in Fig. 5, including an IPC for monitoring the actual position of the robot (IPC1) and another one to perform the feedback loop via wireless communications (IPC2). The LTE air interface is also considered in Fig. 5, including one indoor LTE eNodeB and 2 LTE routers. As the network, that was used to perform these measurements, is deployed in a licensed band, the presence of external interference is almost zero.

Among different settings, we selected three representative scenarios to present in this paper, namely wired connection as a benchmark, LTE under "perfect conditions" and LTE with impairments (low signal-to-noise ratio and other disturbances). Latency value was calculated as a time-stamp difference, between the local position measurement and remote position measurement, respectively. After a latency value was obtained for every point, the mean value was calculated. The numerical 


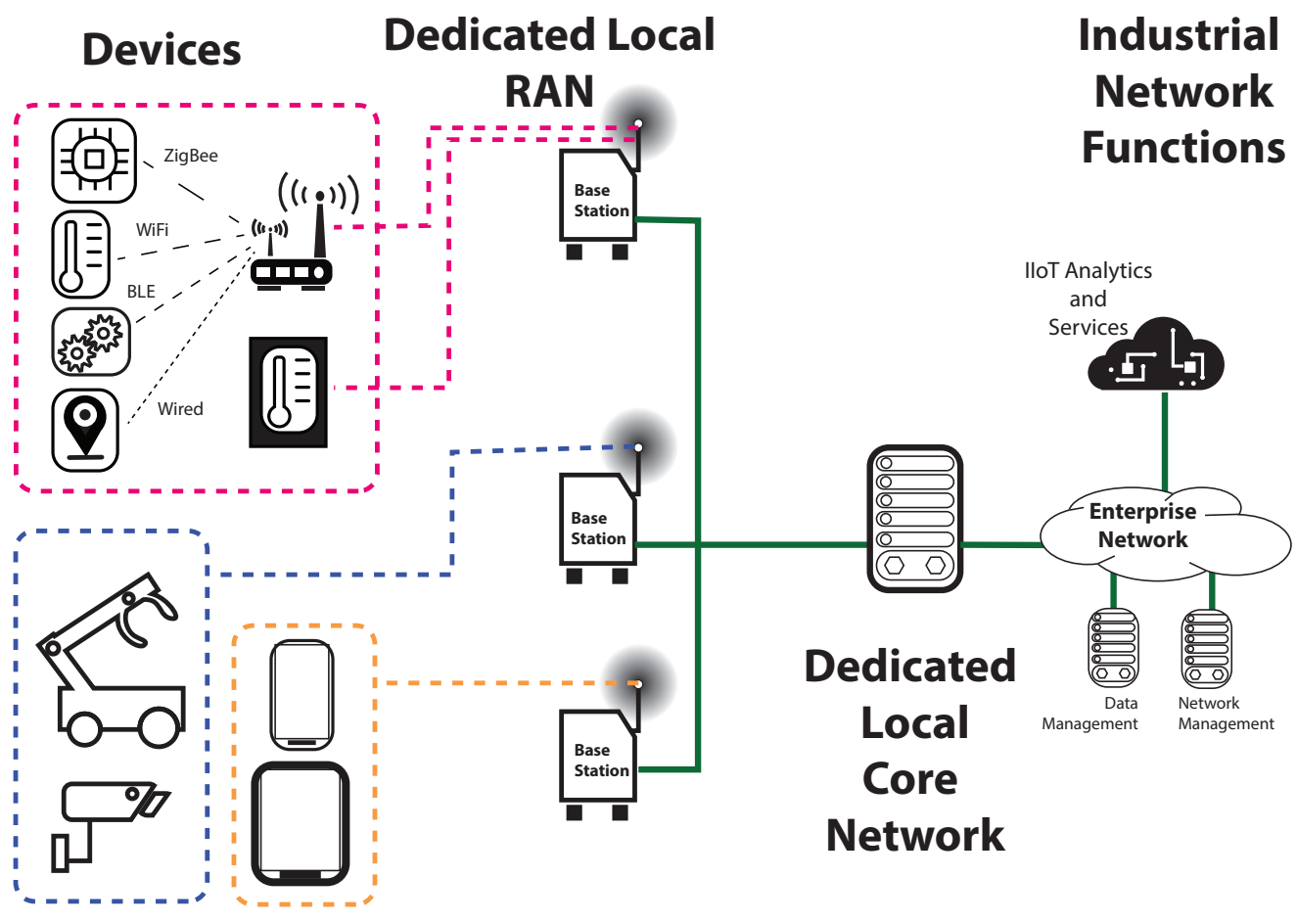

Fig. 3. Factory of the future using a dedicated cellular network for a diversity of industrial applications [19].

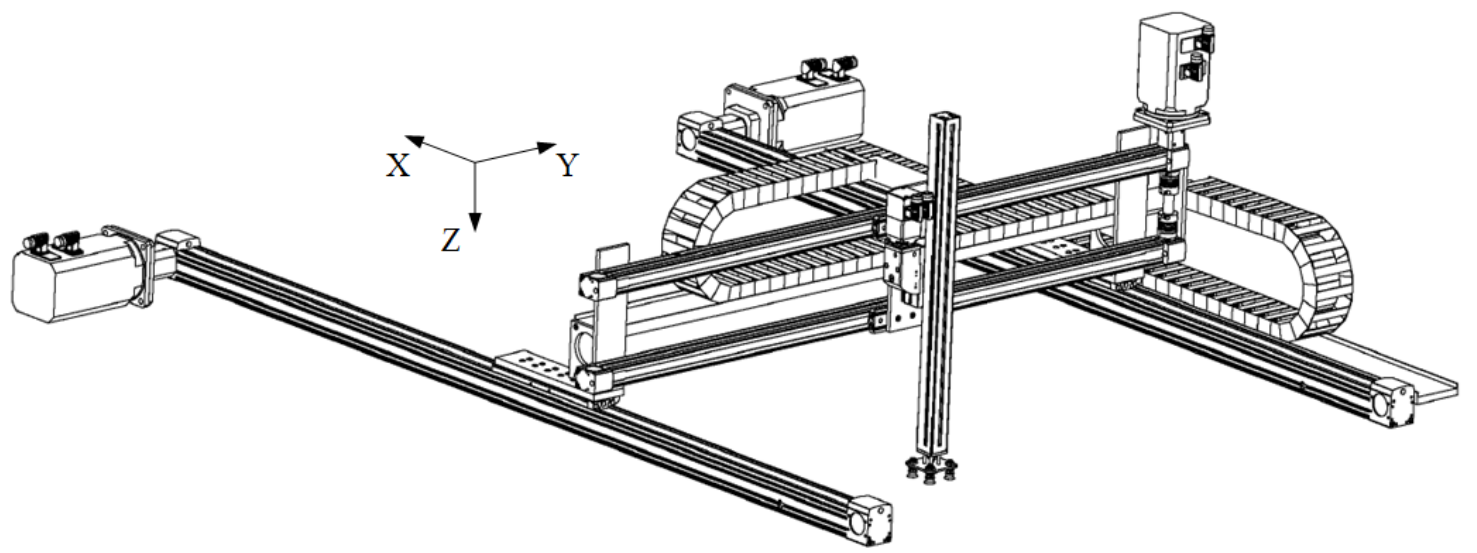

Fig. 4. Position control using a Cartesian robot in $Y$ axis.

results and the details of the experiments carried out are presented in the next section.

\section{NUMERICAL RESULTS}

The description of the experiments carried out here is described next together with an analysis of the numerical results. The proposed testbed to evaluate the different conditions of the LTE network is presented in Fig. 6.

\section{A. Wired network as a benchmark scenario}

In this case a $100 \mathrm{M}$ Ethernet wired interface was used with UDP protocol over Internet Protocol (IP) between first and second IPCs (see Fig. 5). The purpose of this scenario is to obtain a reference mean latency value that is typical for industrial applications. This scenario also represents most of the industrial automation applications, in which the wired connectivity is used instead of wireless. The position feedback of this scenario is presented in Fig.7.

\section{B. LTE network without impairments:}

In this scenario, both LTE routers are positioned in line-ofsight (LOS) with LTE base station. It means that all devices have a direct wireless link between antennas without any obstacle, representing an ideal condition for the wireless channel. As before, UDP is used for transmitting the position measurement. In this scenario, the wireless signal strength; received signal strength indicator (RSSI) was $-51 \mathrm{dBm}$ and signal-tointerference-and-noise ratio (SINR) was $24 \mathrm{~dB}$. These values 


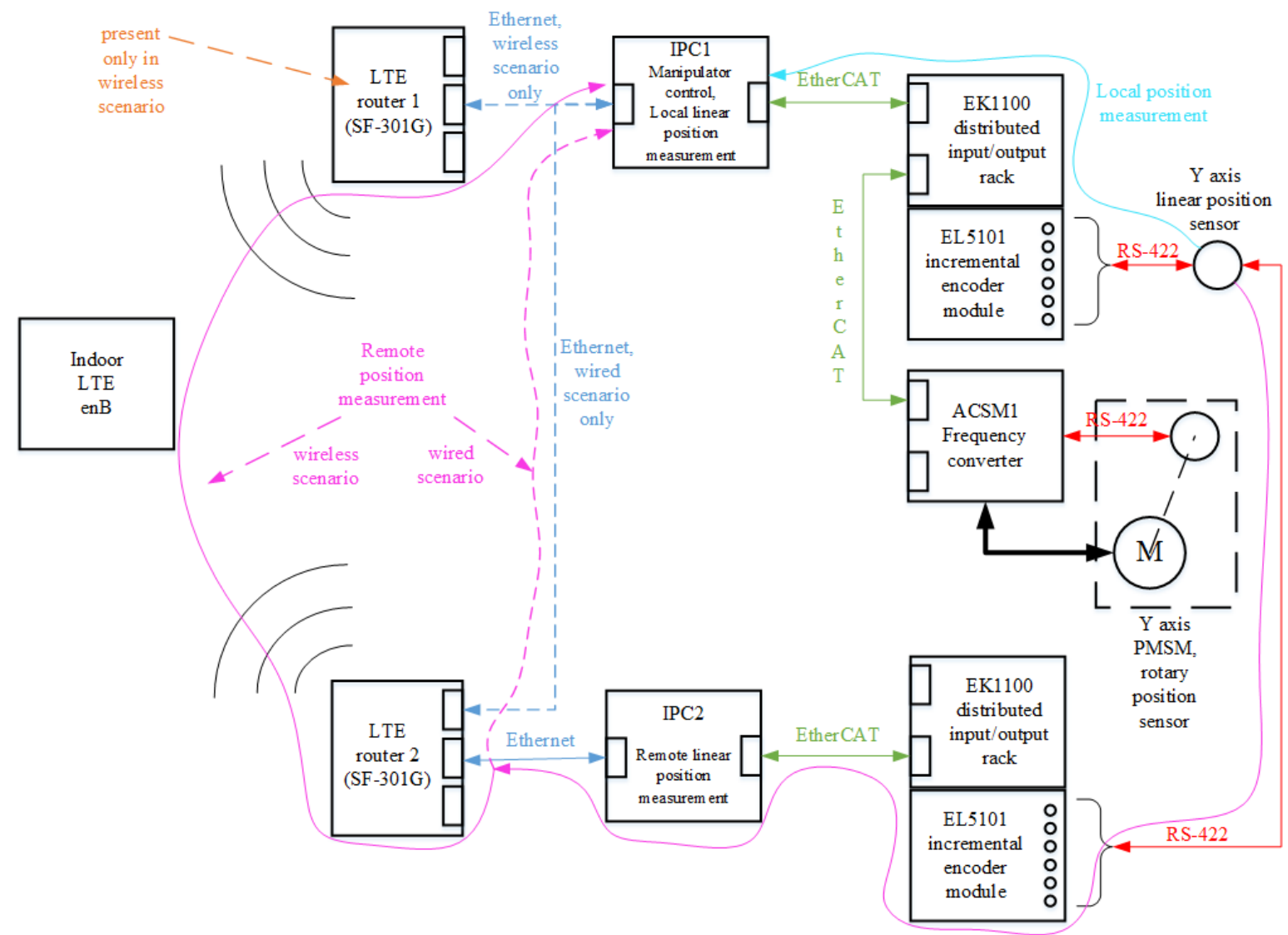

Fig. 5. Test setup functional diagram. Two IPCs are included: one to monitor the actual position, and other to perform the feedback control over LTE.

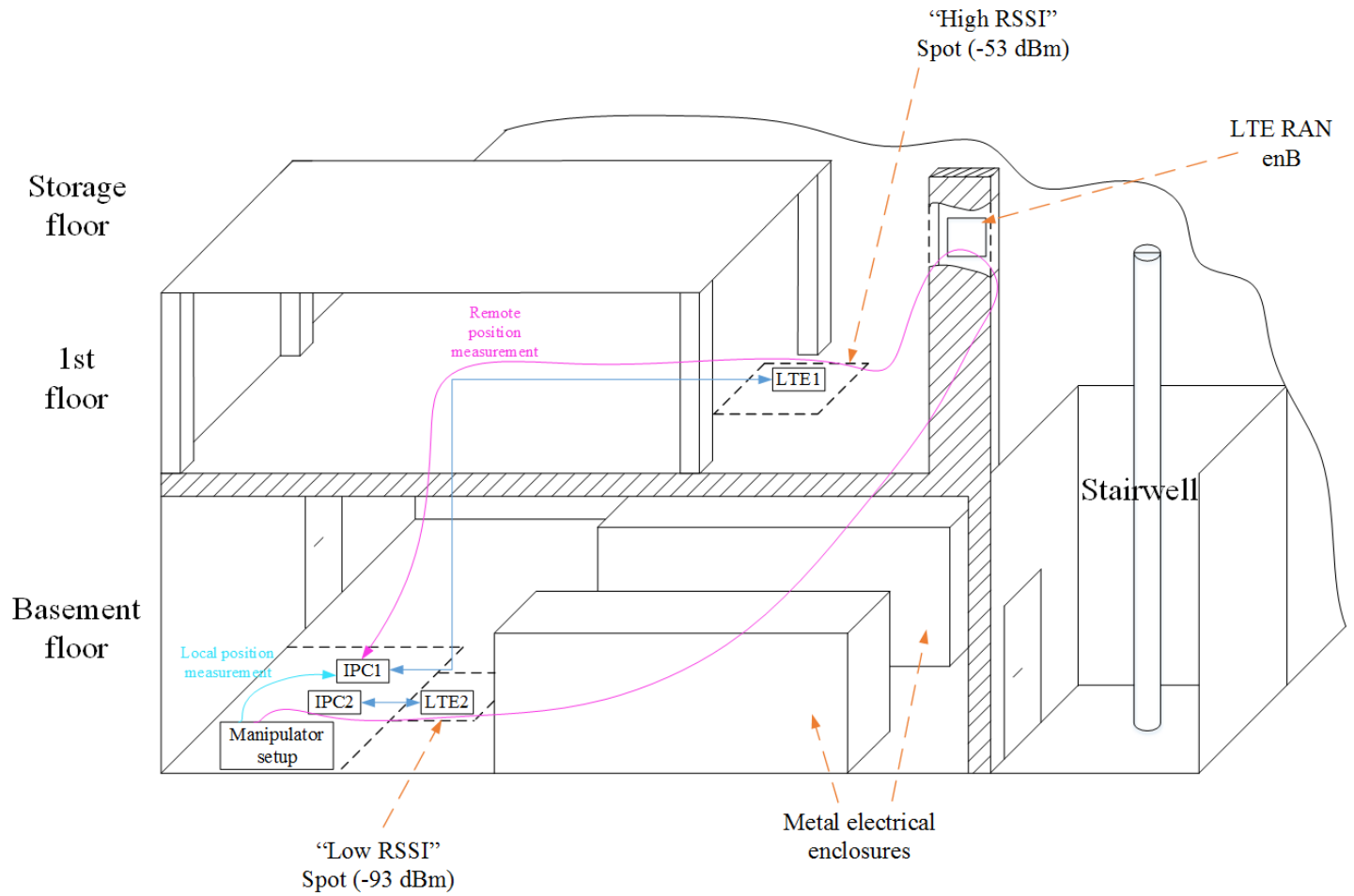

Fig. 6. Device layout for "LTE network with impairments" test scenario. 


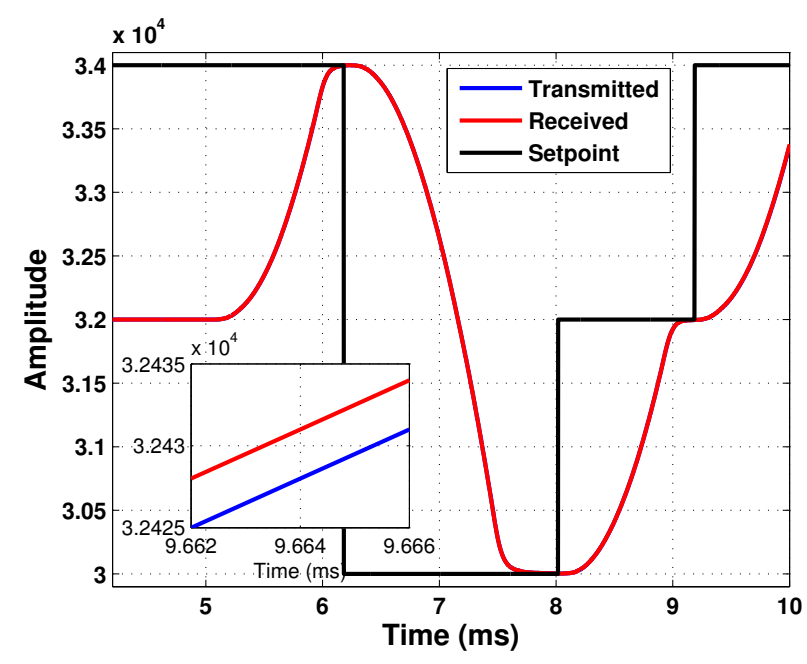

Fig. 7. Wired network as a benchmark, resulting in a mean latency of $3 \mathrm{~ms}$.

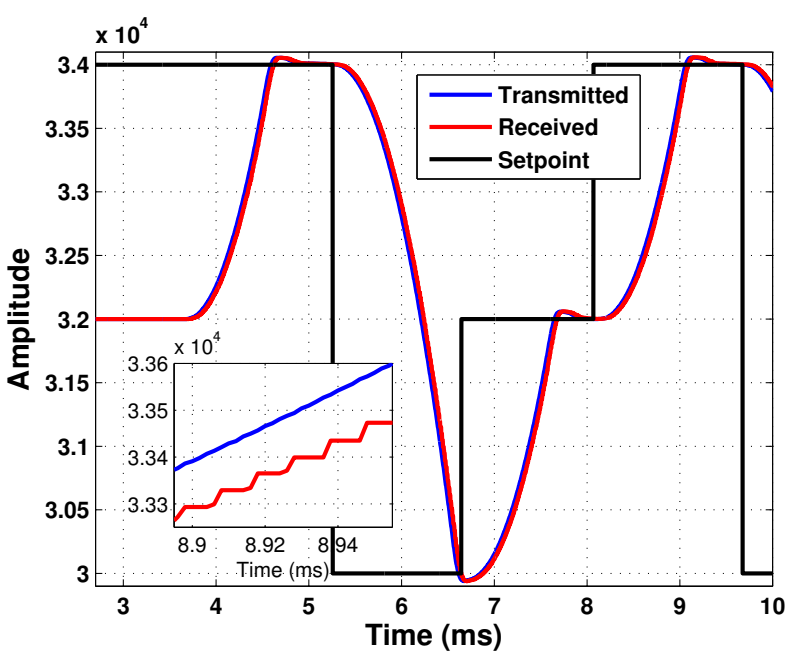

Fig. 8. LTE without impairments, resulting in a mean latency of $26 \mathrm{~ms}$.

are assumed to represent ideal value parameters for a cellular wireless technology. The wireless connectivity is considered between the IPC1 and IPC2 through LTE router 1 and LTE router 2, as depicted in Fig. 5. As it can be noticed from the results in Fig. 8, there is a small position overshoot if compared to the aforementioned reference test case.

\section{LTE network with impairments:}

In order to test a more realistic scenario, some impairments are added to the wireless network presented above. One impairment is related to the network load that is divided in two types, namely uplink load and downlink load. The former is performed by creating an uplink channel congestion between LTE router 2 and LTE base station (eNodeB). It is done by uploading files from a PC connected to LTE router 2 to a cloud storage simultaneously with experiment execution. Downlink load is applied by means of running a video stream in $720 \mathrm{p}$

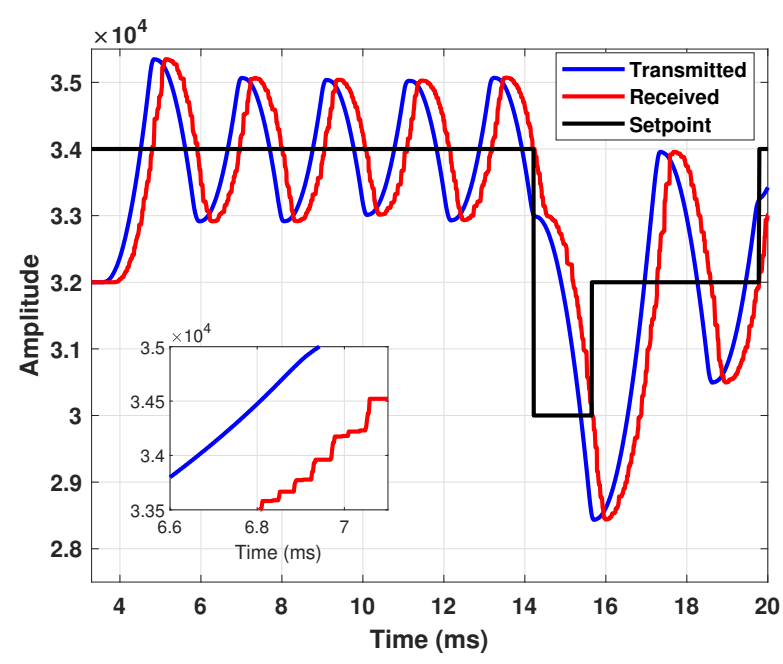

Fig. 9. LTE with impairments, resulting in a mean latency of $290 \mathrm{~ms}$.

on the PC connected to LTE router 1. Another impairment is implemented by inserting an attenuator between LTE router 2 and its antenna to emulate cell border network operation. In addition, LTE router 2 was moved to the basement floor, further decreasing LTE network signal strength. Impairments addition resulted in RSSI value decreasing to $-93 \mathrm{dBm}$ and SINR value to $10 \mathrm{~dB}$, respectively. The Device layout for "LTE network with impairments" scenario is depicted in Fig. 6. Here, it is possible to visualize the physical elements that add impairments in the system - for example, the metal electrical enclosures and the cement material of the 1st floor. Fig. 9 shows an oscillatory behavior of robot arm position, clearly affecting the control task.

\section{Results}

Position setpoint was formed as a sequence of twenty identical cycles, each of them involves three position steps, as presented in Figs. 7, 8, and 9. The First scenario is the benchmark where the feedback loop happens via a $100 \mathrm{M}$ Ethernet cable connection and led to a mean latency of 3 ms. The second scenario presented in Fig. 8 shows a mean latency of $26 \mathrm{~ms}$ when the control loop is performed via a LTE wireless link in ideal conditions; this latency is close to the theoretical value of $20 \mathrm{~ms}$ defined by 3GPP. When the LTE link is deployed to have high attenuation (low RSSI) and faces network congestion, the mean latency is $290 \mathrm{~ms}$, more than ten times worse than in ideal conditions.

\section{CONCLusions}

In this paper a full stack LTE network supporting a specific vertical control application was validated. The wireless network and the cartesian robot used in this study were deployed in a real scenario following industrial guidelines. The real setup was used to provide a clear visualization of how communication latency affects to the automation system, indicating that low-latency required by feedback control loop still cannot be achieved with commercial solutions. For these 
purposes different data traffic was used to increase congestion load in uplink and downlink communication channels.

The latency measurements were done for three specific scenarios. For the benchmark scenario (wired) the latency was estimated to be $3 \mathrm{~ms}$. For the wireless scenario without impairments the latency was equal to $26 \mathrm{~ms}$ as expected. Finally, for the worst case scenario (with impairments) the latency was equal to $290 \mathrm{~ms}$. Therefore, aforementioned experiments support the existing discussions in 3GPP related to machinetype communications under ultra-reliable low-latency regime.

\section{ACKNOWLEDGEMENTS}

This project is partly supported by Academy of Finland via ee-IoT project (ICT2023/n.319009) and via FIREMAN consortium (CHIST-ERA/n.326270).

\section{REFERENCES}

[1] P. Robison, M. Sengupta, and D. Rauch, "Intelligent energy industrial systems 4.0," IT Professional, vol. 17, no. 3, pp. 17-24, May 2015.

[2] M. Aazam, S. Zeadally, and K. A. Harras, "Deploying fog computing in industrial internet of things and industry 4.0," IEEE Transactions on Industrial Informatics, vol. 14, no. 10, pp. 4674-4682, Oct 2018.

[3] E. A. Lee, "Cyber physical systems: Design challenges," in 2008 11th IEEE International Symposium on Object and Component-Oriented Real-Time Distributed Computing (ISORC). IEEE, 2008, pp. 363-369.

[4] A. Hellinger and H. Seeger, "Cyber-physical systems. driving force for innovation in mobility, health, energy and production," Acatech Position Paper, National Academy of Science and Engineering, vol. 1, no. 2 , 2011.

[5] W. Wahlster, "From industry 1.0 to industry 4.0: Towards the 4th industrial revolution," in Forum Business meets Research, 2012.

[6] D. E. Boubiche et al., "Advanced industrial wireless sensor networks and intelligent IoT," IEEE Commun. Mag., vol. 56, no. 2, pp. 14-15, 2018.

[7] D. Lehmann, E. Henriksson, and K. H. Johansson, "Event-triggered model predictive control of discrete-time linear systems subject to disturbances," in 2013 European Control Conference (ECC). IEEE, 2013, pp. 1156-1161.

[8] Y. Cai, Z. Qin, F. Cui, G. Y. Li, and J. A. McCann, "Modulation and multiple access for 5G networks," IEEE Communications Surveys Tutorials, vol. 20, no. 1, pp. 629-646, Firstquarter 2018.

[9] G. A. Akpakwu, B. J. Silva, G. P. Hancke, and A. M. Abu-Mahfouz, "A survey on 5G networks for the internet of things: Communication technologies and challenges," IEEE Access, vol. 6, pp. 3619-3647, 2018.

[10] P. Popovski, J. J. Nielsen, C. Stefanovic, E. d. Carvalho, E. Strom, K. F. Trillingsgaard, A. Bana, D. M. Kim, R. Kotaba, J. Park, and R. B. Sorensen, "Wireless access for ultra-reliable low-latency communication: Principles and building blocks," IEEE Network, vol. 32, no. 2, pp. 16-23, March 2018.

[11] M. Condoluci et al., "Enhanced radio access and data transmission procedures facilitating industry-compliant machine-type communications over LTE-based 5G networks," IEEE Wireless Commun., vol. 23, no. 1, pp. 56-63, 2016.

[12] H. Holma and A. Toskala, LTE for UMTS: Evolution to LTE-advanced. John Wiley \& Sons, 2011.

[13] A. Larmo, M. Lindström, M. Meyer, G. Pelletier, J. Torsner, and H. Wiemann, "The LTE link-layer design," IEEE Communications Magazine, vol. 47, no. 4, pp. 52-59, April 2009.

[14] F. Thiesse, C. Floerkemeier, M. Harrison, F. Michahelles, and C. Roduner, "Technology, standards, and real-world deployments of the EPC network," IEEE Internet Computing, vol. 13, no. 2, pp. 36-43, 2009.

[15] D. Astély, E. Dahlman, A. Furuskär, Y. Jading, M. Lindström, and S. Parkvall, "LTE: the evolution of mobile broadband," IEEE Communications magazine, vol. 47, no. 4, 2009.

[16] 3GPP, "Feasibility study for Further Advancements for E-UTRA (LTE-Advanced) (Release 10) " 3rd Generation Partnership Project (3GPP), Technical Specification (TS) 36.912, 03 2011, version 10.0.0. [Online]. Available: https://portal.3gpp.org/desktopmodules/ Specifications/SpecificationDetails.aspx?specificationId $=2584$
[17] M. Kim, S. Kim, and Y. Lim, "An implementation of downlink asynchronous harq for lte tdd system," in 2012 IEEE Radio and Wireless Symposium, Jan 2012, pp. 271-274.

[18] H. Pai, Y. S. Han, and Y. Chu, "New harq scheme based on decoding of tail-biting convolutional codes in ieee 802.16e," IEEE Transactions on Vehicular Technology, vol. 60, no. 3, pp. 912-918, March 2011.

[19] 3GPP, "Study on Communication for Automation in Vertical Domains (Release 16) ," 3rd Generation Partnership Project (3GPP), Technical Specification Group Services and System Aspects 22.804, 12 2018, version 16.2.0. [Online]. Available: https://portal.3gpp.org/desktopmodules/ Specifications/SpecificationDetails.aspx? specificationId=3187 